\title{
Integrating the organ and tissue allocation processes through an Agent-Mediated Electronic Institution
}

\author{
Javier Vázquez-Salceda $^{1}$, Ulises Cortés ${ }^{1}$, Julian Padget $^{2}$ \\ ${ }^{1}$ Departament de Llenguatges i Sistemes Informàtics. \\ Universitat Politècnica de Catalunya. \\ c/ Jordi Girona 1-3. E08034 Barcelona, Spain. \\ \{jvazquez,ia \}@1si.upc.es \\ Phone: 34934017016 Fax: 34934017014 \\ ${ }^{2}$ Department of Computer Science. University of Bath. Bath, BA2 7AY. United Kingdom. \\ jap@cs.bath.ac.uk \\ Phone 34932919335 Fax 34932919410
}

\begin{abstract}
In this paper we present a first approach to the formalization of Carrel, a virtual organization for the procurement of organs and tissues for transplantation purposes, in order to model the allocation processes of organs and tissues in a integrated way. We show how it can be formalized with the ISLANDER formalism. Also we present a first mechanism to federate the institution in several geographically-distributed platforms.
\end{abstract}

Keywords: Electronic Institutions, Multi-agent systems, Transplants.

\section{Introduction}

Organ transplantation from human donors is the only option available when there is a major damage or malfunction in an organ. At the time of writing, more than one million people in the world have successfully received an organ, and thereafter, in most cases, lead normal lives.

Over the years, transplant techniques have evolved, knowledge of donor-recipient compatibility has improved and so have immunosuppressant drug regimes, leading to an increase in the number of organs that can be transplanted, but also in the range of transplants, moving beyond organs (heart, liver, lungs, kidney, pancreas) to tissues (bones, skin, corneas, tendons). However, the allocation process for tissues is quite different from that for organs, because of the time such pieces can be preserved outside the human body. Tissues are clusters of quite homogeneous cells, so the optimal temperature for preservation of all the cells composing the tissue is almost the same. Thus, tissues can be preserved for several days (from six days in the case of corneas to years in the case of bones) in tissue banks. For tissues, the allocation process is triggered when there is a recipient with a need for a certain tissue, at which time some number of tissue banks are searched for a suitable one. 
Organs, on the other hand, are very complex structures with several kinds of cell types with different optimal preservation temperatures. That fact leads to quite short preservation times (hours), no need for an organ bank, and an allocation process that is triggered when a donor appears, taking the form of a search for a suitable recipient in some number of hospitals.

\subsection{The case for software systems for organ and tissue management}

The increasing rate of success of tissue transplants is leading to an increase in the number of requests. This volume is starting to overwhelm the human coordinators and furthermore is leading to tissue loss, because available tissues are not being assigned due to lack of time to process all requests.

In the case of organs, successful transplants have also led to an increase in demand for organs for transplantation purposes. However, there is not an increasing volume of donations to match the demand. Much research has been done to create policies for donor identification (to increase the number of available donors), organ allocation (to find a suitable recipient for each organ) and in extraction, preservation and implant procedures (to increase the chances of success).

The relative scarcity of (organ) donors has led to the creation of international coalitions of transplant organizations. This new, more geographically distributed, environment makes an even stronger case for the application of distributed software systems to solve:

- the data exchange problem: exchange of information is a major issue, as each of the actors collects different information and stores it in different formats. The obvious, and easily stated, solution is the definition of standard data interchange.

- the communication problem: countries typically use different languages and terminologies to tag the same items or facts. Either a standard notation or a translation mechanism needs to be created to avoid misunderstandings.

- the coordination issues: in order to manage requests at an international level, there is the need to coordinate geographically distributed surgery teams, and to coordinate piece delivery at an international level.

- the variety of regulations: an additional issue is the necessity to accommodate a complex set of, in some cases conflicting, national and international regulations, legislation and protocols governing the exchange of organs. These regulations also change over time, making it essential that the software is adaptable.

The first two points can largely be resolved using standard software solutions. For instance, the EU projects RETRANSPLANT, TECN have devoted most of their effort to the creation of a) standard formats for the storage and exchange of information about pieces, donors and recipients among organizations, b) telematic networks, or c) distributed databases. Another project, ESCULAPE, uses conventional software to help in matching tissue histocompatibility.

The third point (coordination) is harder to solve with conventional software. A sound alternative is the use of software agents, where an Agent is a computer program capable of taking its own decisions with no external control (autonomy), based on its perceptions of the environment and the objectives it aims to reach [18]. It not only reacts to the environment (reactivity) but also proactively takes initiatives. The social ability 
of agents allow them to group together (in agencies) sharing common objectives and dividing the tasks to reach those objectives. All these attributes suggest that multi-agent systems are well-suited to solve coordination issues.

It is the last point (the variety of regulations changing over time) which underpins our case for the use of so-called electronic institutions, whose purpose is to provide over-arching frameworks for agent interaction, where agents may reason about the norms $[6,2,3,5]$, in the same way as physical institutions and social norms do in the real world. Electronic institutions and the norms that govern them are the key to a system that is able to adapt automatically to changes in regulations.

In summary, our proposal address all four issues, by the use of multi-agent technology, not only for coordination and regulation but also serving as a language interface among teams using different terminology, and actively distributing the information to be shared.

\section{An Institution for the distribution of organs and tissues}

The Carrel institution is an agent platform which hosts a group of agents (an agency) responsible for the allocation of organs and tissues. In the case of tissues, the allocation process comprises:

1. The tissue banks keeping the institution updated about tissue availability

2. The agency receiving requests from the hospitals for tissues. For each request (brought by an agent representing the hospital) the institution tries to allocate the best tissue available from all the tissue banks that are known.

In the case of organs, the process comprises:

1. Each hospital informing the institution about patients that have been added to or removed from the waiting list of that hospital, or patients either to be added to or removed from the national-wide Maximum Urgency Level ${ }^{1}$ Waiting List.

2. When a donor appears, the hospital informs the institution of all the organs suitable for donation in the form of offers sent to the organ allocation organization, which then assigns the organs.

Figure 1 depicts all the entities that interact with the Carrel system. There are a) the hospitals that create the tissue requests b) the Tissue Banks, and c) the national organ transplantation organizations, that own the agent platform and act as observers- the figure shows the organizations in Spain: the Organización Nacional de Transplantes ${ }^{2}$ (ONT) [14] and the Organitzaciò CATalana de Transplantaments ${ }^{3}$ (OCATT). In the proposed system all hospitals, even those owning a Tissue Bank, should make their requests for tissues or their organ offers through Carrel in order to ensure a fair distribution of pieces and to ease the tracking of all pieces from extraction to implantation, as the ONT and OCATT currently require for organs.

\subsection{Role of the Carrel Institution}

The role of the Carrel Institution can be summarized by the following tasks:

\footnotetext{
${ }^{1}$ In Spain the Maximum Urgency Level is called Urgency-0

${ }^{2}$ National Transplant Organization

${ }^{3}$ Catalan Transplant Organization
} 


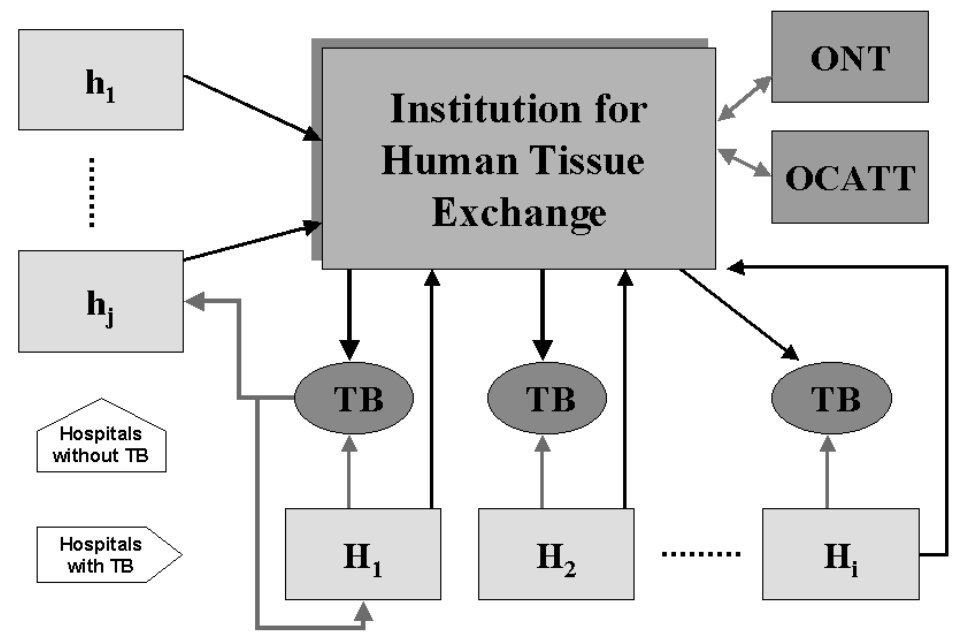

Fig. 1. Carrel: An Agent Mediated Institution for Tissues Assignment

- to make sure that all the agents which enter into the institution behave properly (that is, that they follow the behavioral norms).

- to be up to date about all the available pieces in the Tissue Banks, and all the recipients that are registered in the waiting lists.

- to check that all hospitals and tissue banks fulfill all the requirements needed to interact with Carrel.

- to take care of the fulfillment of the commitments undertaken inside the Carrel system.

- to coordinate the piece delivery from one facility to another.

- to register all incidents relating to a particular piece.

\subsection{The UCTx Agency}

The participation of hospitals in Carrel is based on the notion of membership. That is, hospitals belong to the Institution and respect the negotiation (assignation) rules, and the agents that represent them inside Carrel are unable to break these conventions. A Hospital interacts with Carrel through the Transplant Coordination Unit Agency (UCTX). A version of the UCTx agent architecture that handles tissue requests can be found in [4].

Adapting the UCTx agency in order to assist not only in the tissue allocation process but also in the organ allocation process is not difficult. In the case of tissues, it is surgeons who are responsible for creating the tissue requests through their Surgeon Agent [4]. In contrast, for organs it is the Hospital Transplant Coordinator who is responsible for issuing organ offers to the institution or answering a call for recipients. So the architecture presented in [4] does not need to be modified but instead just the functionality of the Coordinator Agent is extended. 


\section{Formalizing the Carrel institution}

To give a formal description of the interaction among agents in the Carrel system we will follow the ISLANDER formalism [7]. It views an agent-based electronic institution as a type of dialogical system where all the interactions inside the institution are a composition of multiple dialogic activities (message exchanges). These interactions (called illocutions [13]) are structured through agent group meetings called scenes that follow well-defined protocols.

\subsection{The performative structure}

The connected graph of scenes constitutes the performative structure. It is a network of scenes that defines the possible paths for each agent role. In accordance with its role, an agent may or may not be permitted to follow a particular path through the performative structure, and ultimately, may be required to leave the institution.

In the case of the Carrel institution, the set of scenes to model the organ and tissue allocation processes is:

- Reception Room: is the scene where all external agents should identify themselves in order to be assigned the roles they are authorized to play. If these agents carry either a request for one or more tissues or an offer of one or more organs, then this information is checked to make sure that it is well-formed.

- Consultation Room: is the scene where the institution is updated about any event or incident related to a piece. Agents coming from tissue banks should update the institution about tissue availability, while agents coming from hospitals should update the institution about the waiting lists and also inform it about the reception of all pieces (organs or tissues) they have received, the transplant operation and the condition of recipients.

- Exchange Room: is the scene where assignation of pieces takes place. There are specific exchange rooms for tissue requests (Tissue Exchange Room) and for organ offers (Organ Exchange Room).

- Confirmation Room: is the scene where the provisional assignments made in one of the exchange rooms are confirmed, whereafter a delivery plan is constructed, or cancelled, because a new request of higher priority has arrived.

A key element of the ISLANDER formalism is the definition of agent roles. Each agent can be associated to one or more roles, and these roles determine the scenes an agent can enter and the protocols it should follow (the scene protocols are defined as multi-role conversational patterns). There are two kinds of roles: the external roles (roles for incoming agents) and the institutional roles (roles for agents that carry out the management of the institution). The external roles are the following:

Hospital Finder Agent (hf): agents sent by hospitals with tissue requests or organ offers that are seen from the point of view of the institution as requests for finding an acceptable tissue or recipient, respectively.

Hospital Contact Agent (hc): agents from a certain hospital that are contacted by the institution when an organ has appeared for a recipient on the waiting list of that hospital. The agent then enters the institution to accept the organ and to receive the delivery plan. 


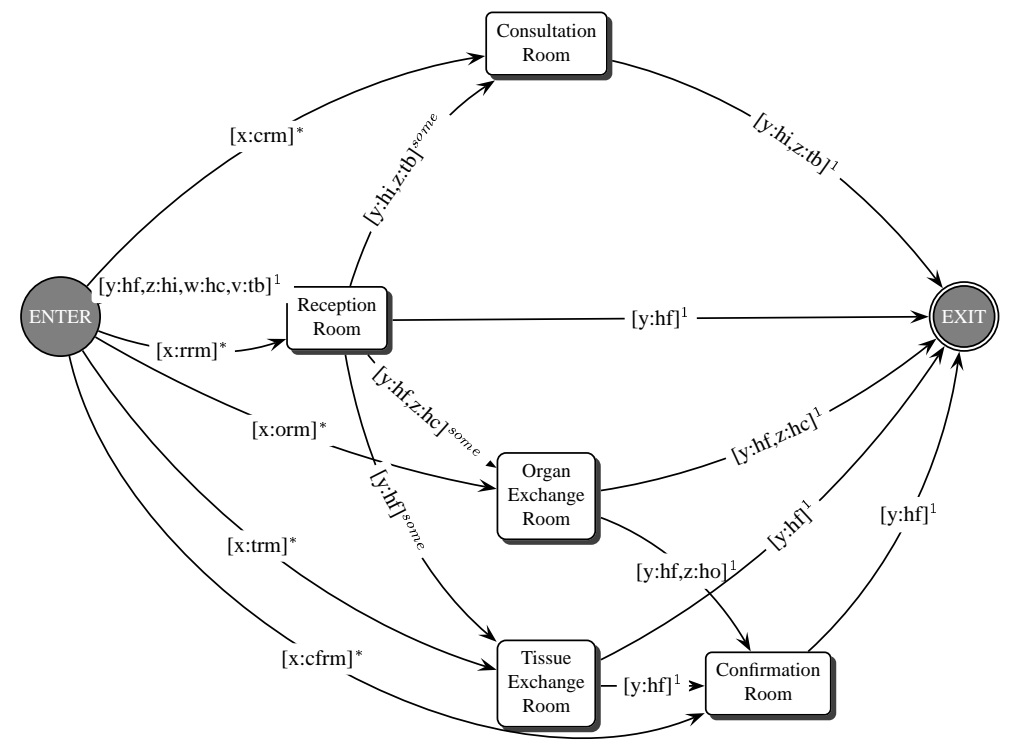

Fig. 2. The Carrel Institution performative structure

Hospital Information Agent (hi): agents sent by hospitals to keep the Carrel system updated about any event related to a piece or the state of the waiting lists. They can also perform queries on the Carrel database through the DB Agent (see $\S 3.2$ ).

Tissue bank notifier (tb): agents sent by tissue banks in order to update Carrel about tissue availability.

The institutional roles consist of one agent to manage each scene and one agent to coordinate all the scene relationships:

Institution Manager (im): agent coordinating all the scene managers.

Reception Room Manager (rrm): manager of the Reception Room scene.

Tissue Exchange Room Manager (trm): manager of a Tissue Exchange Room scene. Organ Exchange Room Manager (orm): manager of a Organ Exchange Room scene. Confirmation Room Manager (cfrm): manager of the Confirmation Room scene.

Consultation Room Manager (crm): manager of the Consultation Room scene.

With all the scenes and roles identified in the previous section, the performative structure can be drawn, as depicted in figure 2. Nodes are the scenes listed above plus enter and exit nodes which define the begin and end points of the diagram. Arcs are labelled with tags variable:role, where variable is an agent ${ }_{i}$ and role is one among the identified Carrel's roles. The diagram in figure 2 shows, for instance, that scene's managers go directly from the enter point to the scene they should manage (the $*$ means that they are the ones creating the scene), while all the external agents must proceed 
through the Reception Room scene in order to be registered and then be directed to the proper scene according to their roles.

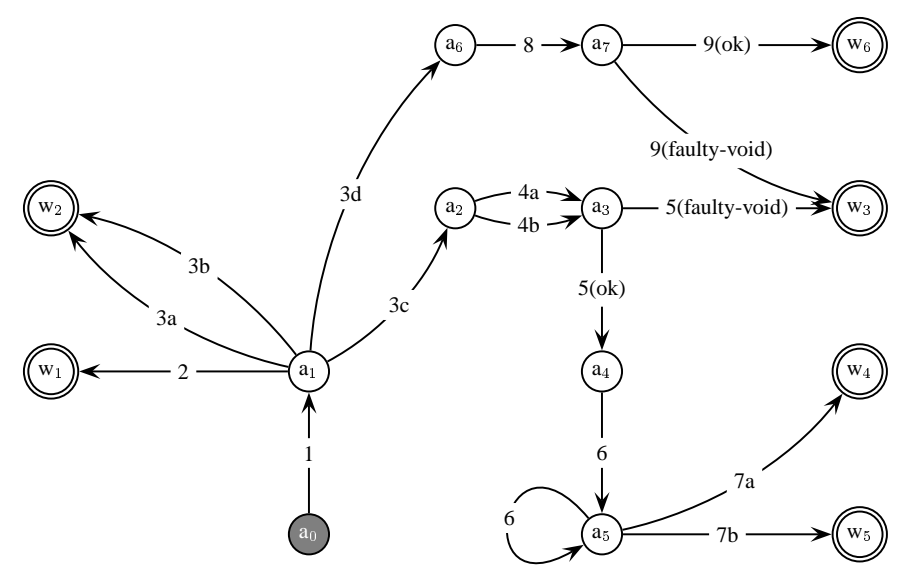

Fig. 3. The conversation graph for the Reception room

Authentication of external agents As explained above, in the Reception Room external agents enter and are registered inside the platform. In this room an authentication mechanism based in electronic certificates ensures that external agents come only from authorized organizations (which previously received the electronic certificate to be used). Once the sender has been identified and authorized, the external agents are then directed to the proper room according to their roles.

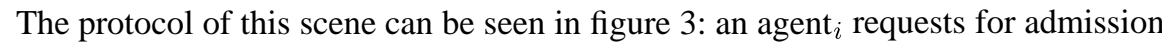
(1) and may be accepted (messages $3 \mathrm{a}, 3 \mathrm{~b}, 3 \mathrm{c}, 3 \mathrm{~d}$ ) or refused (message 2, exit state $w_{1}$ ). According to the role of the incoming agent : $_{\text {: }}$

- it is headed to the Consultation Room (exit state $w_{2}$ ),

- if it brings a request from a hospital, the request is checked (messages 4 and 5). Then agent ${ }_{i}$ waits until the appropriate Exchange Room is available for the assignation (messages 6 and $7 \mathrm{a}$ for tissues, 6 and $7 \mathrm{~b}$ for organs).

- if it was called by the institution to receive an organ offer, the information it brings about the recipient is checked and, if all is correct, it is then directed to the Organ Exchange Room that sent the call.

The content of the messages that appear in this conversation graph and the following ones are specified in [17].

Registering the recipients and the available pieces In order to manage the assignation of organs and tissues, the Carrel institution needs up to date information on a) all the available tissues for transplantation, b) the state of hospitals waiting list for each kind of organ, and c) the whereabouts about all pieces that have been assigned by Carrel. 


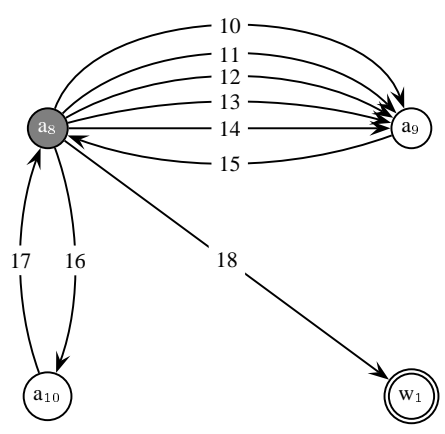

Fig. 4. Conversation graph for the Consultation room

The Consultation Room allows agents coming from hospitals or tissue banks to keep Carrel updated about all the facts mentioned above. The protocol of this scene is shown in figure 4 . The incoming agents can perform notifications (messages 10 to 14) and are informed if the notification is successful (message 15). The agents coming from hospitals — which represent the Hospital Transplant Coordinator [4] — can also perform queries (message 16) about historical facts (e.g. statistics on, say, successful cornea transplantations over a certain period). The queries are answered (message 17) with the level of detail that is permitted for a certain role, as all access to the database is controlled through a Role-Based Access Model [10]. When the incoming agents have performed all the queries and notifications, they exit the Carrel system (message 18).

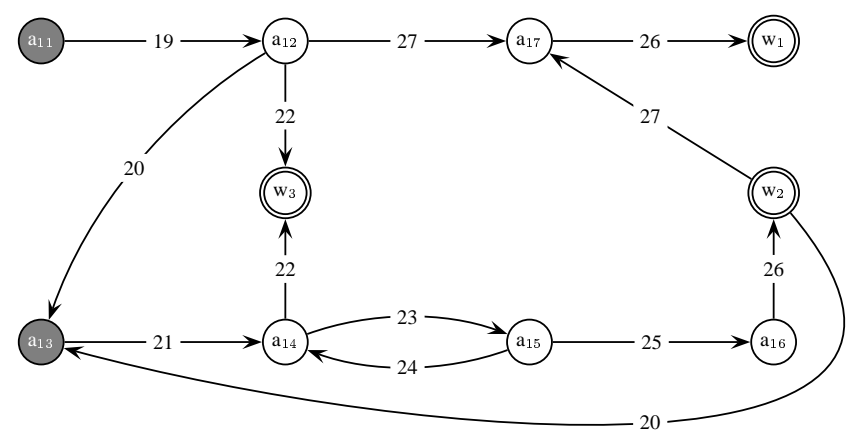

Fig. 5. The conversation graph for the Organ Exchange room

Allocating organs For organ assignment, a new scene, the Organ Exchange Room has been added. The protocol of this scene, depicted in figure 5, can be divided in two parts:

- the arrival of an Agent ${ }_{i}$ (hospital Finder Agent) with an offer of an available organ (states $a_{11}$ and $a_{12}$ ), waiting for a notification that a proper recipient has been found 
(message 22, exit state $w_{3}$ ) or not (message 27 leading to an exit request through state $\left.w_{1}\right)$.

- the loop of the scene manager looking for recipients. Based on the information of the waiting lists stored in Carrel's database, the scene manager sends a call to a hospital (message 20) where there is a suitable recipient. Then an Agent ${ }_{j}$ (hospital Contact Agent) enters the scene to answer the call, saying whether it accepts the organ or not (message 20). Sometimes Agent ${ }_{j}$, representing the hospital Transplant Coordinator, expresses the intention to use the organ in a different recipient (message 23), a change that, depending on the reasons given, can either be accepted or rejected (messages 24 and 25). If the scene manager and Agent ${ }_{j}$ agree, then Agent $_{i}$ is notified of the recipient, otherwise Agent $_{j}$ exits the scene and the loop starts again with a call to another hospital for another recipient.

The search and assignment processes by the scene manager are driven by knowledge of donor-recipient compatibility that is coded in the form of rules such as the following ones for kidneys:

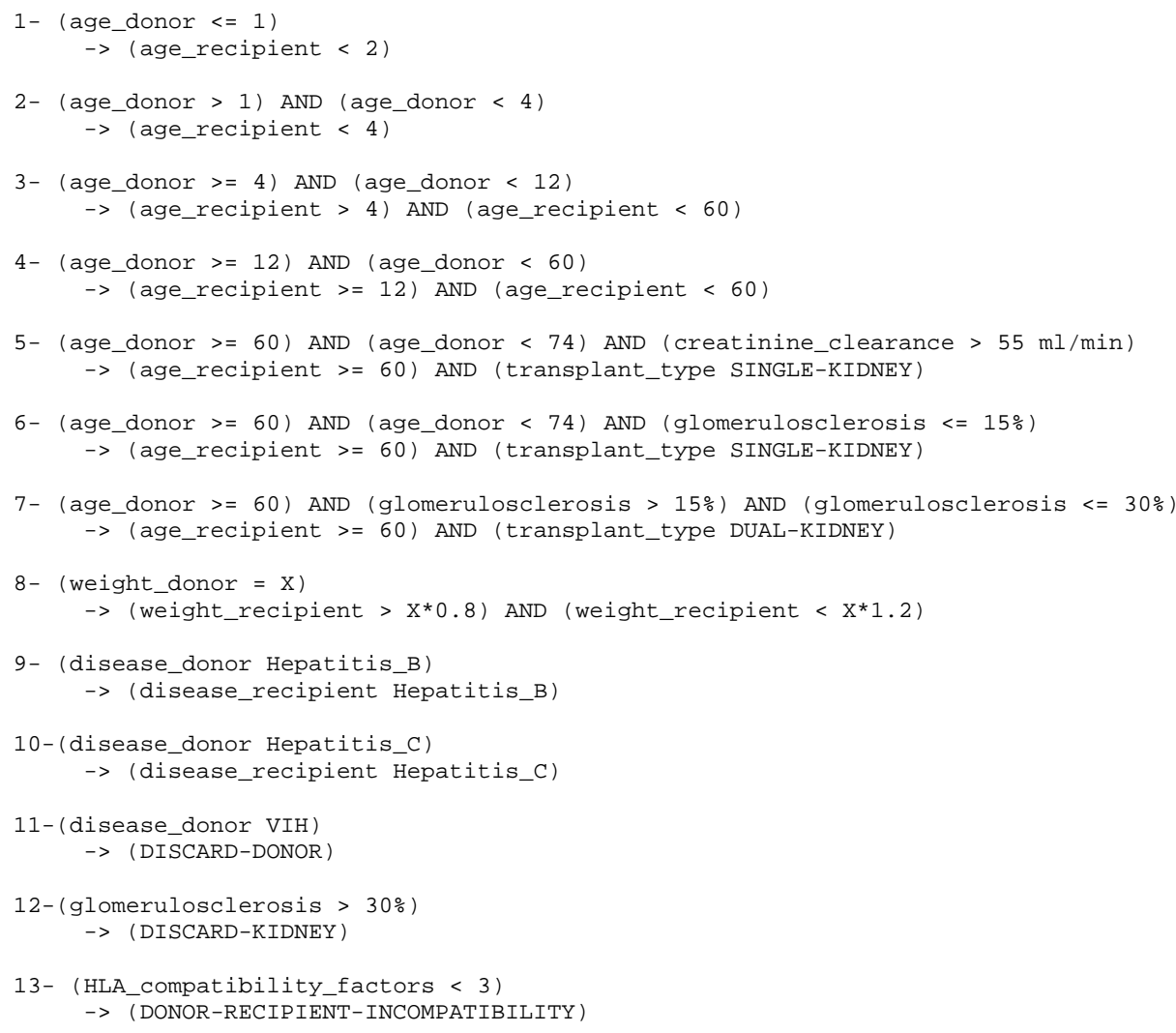

Rules 1 to 8 are related to size compatibility, either considering age ranges (rules 1 to 7) or weight differences, here the criterion permits a $20 \%$ variation above or below. Rules 5 to 7 consider quality of the kidney and assess not only the limit that is acceptable 
but also the transplant technique to be used (to transplant one or both kidneys). Rules 9 to 10 are examples of diseases in the donor that do not lead to discarding the organ for transplantation, if a proper recipient is found (in the example, a recipient that has had also the same kind of hepatitis B or C in the past). Finally, rules 11 to 13 are examples of rejection rules, as determined by current medical knowledge.

It is important that such policies not be hard-coded in the system, as such rules evolve with practice (for instance, some years ago donors with any kind of Hepatitis were discarded). Expressing the knowledge in the form of rules is a technique that allows the system to be adaptable to future changes in medical practice.

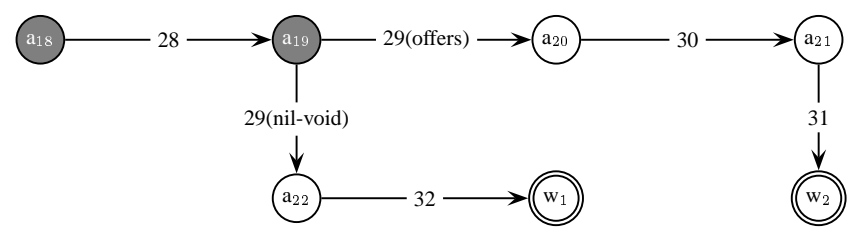

Fig. 6. The conversation graph for the Tissue Exchange room

\begin{abstract}
Allocating tissues The Tissue Exchange Room is the place where negotiation over tissues is performed. The protocol of this scene is shown in figure 6: Agent (hospital Finder Agent) asks the scene manager for tissue offers (tissues matching the requirements included in their petition). Then the scene manager gives a list of available tissues (message 29) that is evaluated by the external agent $i$ (message 30). With this information the scene manager can make a provisional assignment and solve collisions (two agents interested in the same tissue). When this provisional assignment is delivered (message 31) then agent $i_{i}$ exits the scene to go to the Confirmation Room represented by state $w_{2}$. There is an alternative path for the case when there are no available pieces matching the requirements described in the petition (message 9 with null list). In this case agent ${ }_{i}$ requests an exit permission from the institution (message 32, exit state $w_{1}$ ), including the reason for leaving. The reason provided is recorded in the institution logs to form an audit trail for the relevant authorities to inspect. For further information about this negotiation process see [16].
\end{abstract}

Confirming the assignation In the Confirmation Room scene, the provisional assignments made in a Tissue Exchange Room or an Organ Exchange Room are either confirmed or withdrawn. Figure 7 shows the protocol of this scene: the agent $t_{i}$ can analyze the assigned piece data and then accept or refuse it (message 33). If the agent accepts the piece and no higher-priority requests appear during a certain time window then the provisional assignment is confirmed and a delivery plan is given to the agent (message 34 ), and then it exits the Carrel system (exit state $w_{2}$ ). When there is a request with higher priority that needs the piece provisionally assigned to agent ${ }_{i}$ a conflict arises. To resolve the conflict the scene manager notifies the agent ${ }_{i}$ that the assignment has been 


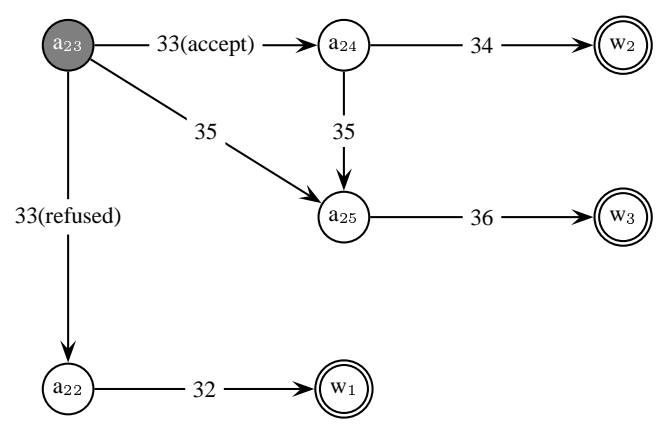

Fig. 7. Conversation graph for the Confirmation room

withdrawn (message 35) and that he is then entitled to a fresh request for another piece, if available, (message 36) to be negotiated again in the Exchange Room whence it came.

\subsection{The Multi-agent architecture}

The agent architecture that performs the institutional roles is shown in figure 8 . There is one agent managing each of the scenes: the RR Agent managing the Reception Room, the CR Agent managing the Consultation Room, an ER Agent for each Exchange Room (either the ones for organs or the ones for tissues), and a CfR Agent managing the Confirmation Room. Also there is an agent (the IM Agent) playing the institution manager role.

In order to assist those agents, two agents are added for specific tasks: the Planner Agent, to build the delivery plans that are needed in the Confirmation Room, and the DB Agent, which is devoted to the role-based access control of the internal Database.

\section{A network of Carrel institutions}

In the previous sections the Carrel system has been described as an institution that works alone, managing all the requests and offers coming from the hospitals. However a distributed system is needed in order to manage the allocation problem at an international level (one of the aims of our scheme).

To do so, we propose to create a federation of geographically-distributed Carrel platforms. Hospitals and Tissue banks register themselves to the "nearest" platform and interact as described in previous sections.

As a result, the search process is distributed through the platforms, exchanging information among themselves via their DB Agents. The process is the following:

- The DB Agent of a certain platform ${ }_{i}$ receives a query, either from an Organ Exchange Room, a Tissue Exchange Room or the Consultation Room

- It accesses the local database.

- If the information is not available locally, then it sends part of the query to other DB Agents in other Carrel Platforms. 


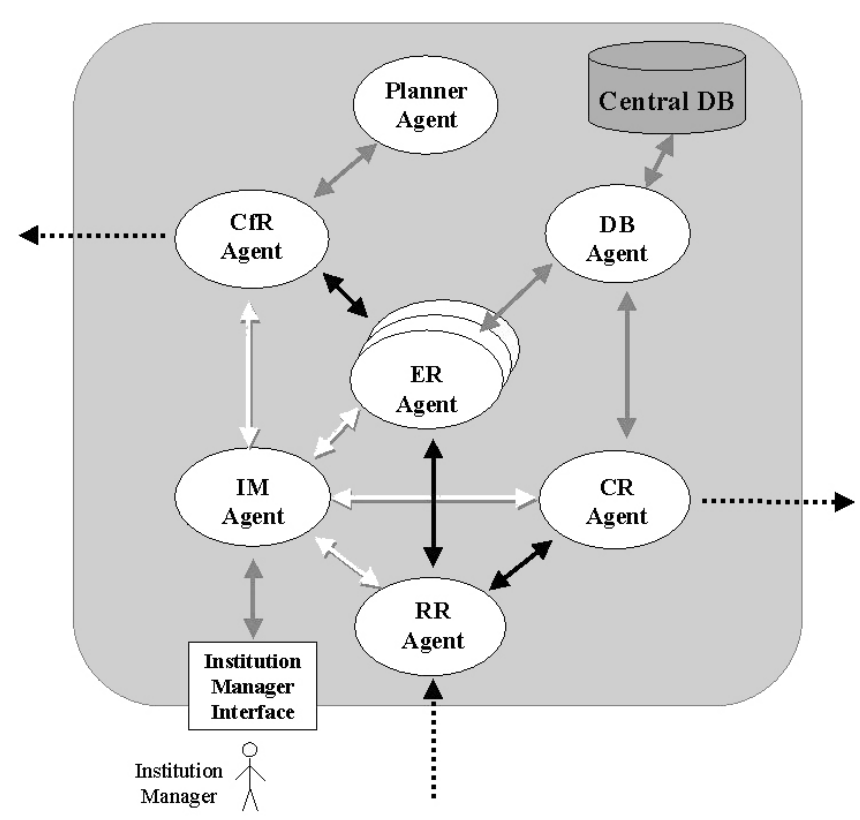

Fig. 8. The multi-agent architecture of a Carrel platform

- All the diferences in terminology are solved at this point by the use of domain ontologies shared by all the platforms that define a common exchange format for the information.

All Carrel platforms are aware of the existence of the other platforms. The communication among agents on different platforms is acheived by the mechanism defined in the FIPA specification for communication among Agent platforms [8].

\section{Conclusion}

We have presented here an Agent-Mediated Electronic Institution for the distribution of organs and tissues for transplantation purposes. Our aim with this work is not only to apply multi-agent technologies to model the organ and tissue allocation problem but we also have devoted part of our efforts in formalization, following the recommendations in [9] about the need of formal design methods when applying agents to the medical domain in order to ensure the safety and soundness of the resulting system. In our case we have chosen a formalism called ISLANDER [7], based on the dialogical framework idea, to get an accurate description of the interactions among the agents. By means of such formalism we have been able to design a system that combines the strengths of agents with the adventages of formal specifications.

As far as we know, there are very few references in the literature about the use of agents in the transplant domain. [15] and [12] describe single agents to solve spe- 
cific tasks needed for this domain (respectively, a receiver selection algorithm based on multi-criteria decision techniques and a planner for transport routes between hospitals for organ delivery). [11] proposes a multi-agent system architecture to coordinate all the hospital members involved in a transplant. [1] also proposes a static hierarchical agent architecture for the organ allocation problem, but no formalism is used in the development of the architecture, and no mechanism is presented to make the architecture adaptive to changes in policies or regulations. For an extended discussion see [16].

Future work aims to extend the methodology to introduce explicit representations of norms to allow agents to reason about the norms. The agents will be able to make better choices in special circumstances. We will follow Dignum's work in [6], incorporating the abstract norms and values of real organizations' statutes and formally connecting them with their implementation in the electronic institution's procedures and protocols. In doing so we will get a full description of an institution, from the abstract (higher) level to the implementation (lower) one.

\section{Acknowledgements}

U. Cortés, and J. Vázquez-Salceda want to acknowledge the IST-2000-28385 Agentcities.NET and the IST-1999-10176 A-TEAM projects. The views in this paper are not necessarily those of Agentcities.NET and A-TEAM consortia.

\section{References}

1. A. Aldea, B. López, A. Moreno, D. Riaño, and A. Valls. A multi-agent system for organ transplant co-ordination. In Barahona Quaglini and Andreassen (Eds.), editors, Proceedings of the 8th. European Conference on Artificial Intelligence in Medicine, Portugal, 2001., Lecture Notes in Artificial Intelligence 2101: Artificial Intelligence in Medicine, pages 413416., 2001.

2. C. Castelfranchi, F. Dignum, C. Jonker, and J. Treur. Deliberate normative agents: Principles and architecture, 1999.

3. R. Conte and C. Castelfranchi. Are incentives good enough to achieve(info)social order? In V. Shankararaman, editor, Workshop on Norms and Institutions in Multi-Agent Systems. ACM-AAAI, ACM Press, 2000.

4. U. Cortés, J. Vázquez-Salceda, A. López-Navidad, and F. Caballero. UCTx: A multi-agent system to assist a transplant coordinator unit. Applied Intelligence. Accepted, 2002.

5. C. Dellarocas and M. Klein. Contractual Agent Societies: Negotiated shared context and social control in open multi-agent systems. In V. Shankararaman, editor, Workshop on Norms and Institutions in Multi-Agent Systems, pages 1-11, 2000.

6. F. Dignum. Abstract norms and electronic institutions. In Proceedings of the International Workshop on Regulated Agent-Based Social Systems: Theories and Applications (RASTA '02), Bologna, 2002.

7. Marc Esteva, Julian Padget, and Carles Sierra. Formalizing a language for institutions and norms. In Milinde Tambe and Jean-Jules Meyer, editors, Intelligent Agents VIII, Lecture Notes in Artificial Intelligence. Springer Verlag, 2001. To appear.

8. The Foundation for Intelligent Phisical Agents, http://www.fipa.org/repository/fipa2000.html. FIPA Specifications, 2000.

9. J. Fox and S. Das. Safe and Sound. MIT Press, $1^{\text {st }}$ edition, 1999. 
10. A. Lin. Integrating policy-driven role-based access control with common data secutiry architecture. Technical Report HPL-1999-59, HP Laboratories Bristol, 1999.

11. A. Moreno, A. Valls, and J. Bocio. Management of hospital teams for organ transplants using multi-agent systems. In Barahona Quaglini and Andreassen (Eds.), editors, Proceedings of the 8th. European Conference on Artificial Intelligence in Medicine, Portugal, 2001, Lecture Notes in Artificial Intelligence 2101: Artificial Intelligence in Medicine, pages 374-383, 2001.

12. A. Moreno, A. Valls, and A. Ribes. Finding efficient organ transport routes using multi-agent systems. In IEEE 3rd International Workshop on Enterprise Networking and Computing in Health Care Industry (Healtcom), L'Aquilla, Italy, 2001.

13. P. Noriega. Agent-Mediated Auctions: The Fishmarket Metaphor. Number 8 in IIIA Monograph Series. Institut d'Investigació en Intel.ligència Artificial (IIIA), 1997. PhD Thesis.

14. Organización Nacional de Transplantes. http://www.msc.es/ont.

15. A. Valls, A. Moreno, and D. Sánchez. A multi-criteria decision aid agent applied to the selection of the best receiver in a transplant. In 4th. International Conference on Enterprise Information Systems, Ciudad Real, Spain, 2002.

16. J. Vázquez-Salceda, U. Cortés, and J. Padget. Formalizing an electronic institution for the distribution of human tissues. In Artificial Intelligence in Medicine. Accepted, 2002.

17. J. Vázquez-Salceda, U. Cortés, J. Padget, A. López-Navidad, and F. Caballero. Extending the carrel system to mediate in the organ and tissue allocation processes: A first approach. Technical Report LSI-02-32-R, Departament de Llenguatges i Sistemes Informàtics, 2002.

18. M. Wooldrige and N.R. Jennings. Intelligent agents: Theory and practice. The Knowledge Engineering Review, 10(2):115-152, 1995. 